\begin{tabular}{lccr} 
QUARTERLY & OF & APPLIED & MATHEMATICS \\
\hline Vol. III & JULY, 1945 & No. 2 \\
\hline
\end{tabular}

\title{
SOME NUMERICAL METHODS FOR LOCATING ROOTS OF POLYNOMIALS*
}

\author{
BY \\ THORNTON C. FRY \\ Bell Telephone Laboratories
}

1. Introduction. It is the purpose of this paper to discuss the location of the roots of polynomials of high degree, with particular reference to the case of complex roots. This is a problem with which we at the Laboratories have been much concerned in recent years because of the fact that the problem arises rather frequently in the design of electrical networks. I shall not give any attention to strictly theoretical methods, such as the exact solution by elliptic or automorphic functions: nor to the development of roots in series or in continued fractions, though such methods exist and one at least-development of the coefficients of a quadratic factor ${ }^{1-i s}$ of great value in improving the accuracy of roots once they are known with reasonable approximation.

Instead, we shall deal with just two categories of solutions: one, the solution of the equations by a succession of rational operations, having for their purpose the dispersion of the roots; the other, a method depending on Cauchy's theorem regarding the number of roots within a closed contour.

\section{PART I-MATRIX ITERATION}

2. Duncan and Collar. We shall treat the first category by a method recently elaborated by Duncan and Collar in two papers in the Philosophical Magazine. ${ }^{2}$ I do not know how thoroughly these writers appreciate the close relationship of their work to that of the other writers whom I shall mention in the course of my presentation. The fact that their interest was primarily concerned with certain broad dynamical problems may perhaps have inhibited them from taking some of the steps which I shall take in their name. But they at least possessed the essential idea, and exhibited quite sufficient ability in the development of it to warrant the assertion that my presentation only differs from theirs in detail-sometimes details of omission, sometimes details of amplification.

* Received Dec. 26, 1944.

1 The essence of this method is contained in a section of Legendre's Essai sur la therorie des nombres. It is also attributed to Bairstow by Frazer and Duncan. It was developed independently, and perhaps somewhat more fully, by the present writer; but the extensions seem so obvious that it has not appeared to warrant separate publication.

${ }^{2}$ W. J. Duncan and A. R. Collar, A method for the solution of oscillation problems by matrices, Phil. Mag. (7) 17, 865-909 (1934); Matrices applied to the motions of damped systems, Phil. Mag. (7) 19, 197-219 (1935). 
3. The fundamental identity. We begin by noting that the $\lambda$-determinant

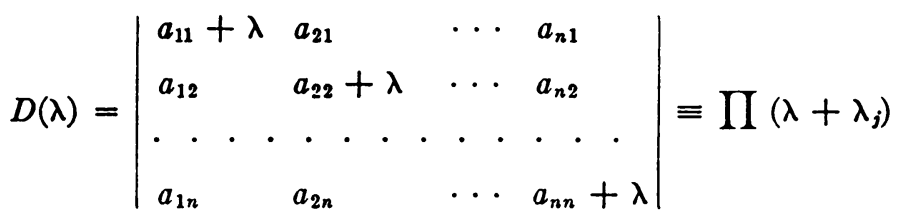

is the characteristic function ${ }^{3}$ of the matrix

$$
M=\left\|a_{i j}\right\|
$$

and its determinant

$$
\Delta=\left|a_{i j}\right| .
$$

It is obviously a polynomial of degree $n$, which we may write

$$
D(\lambda)=\lambda^{n}+p_{1} \lambda^{n-1}-p_{2} \lambda^{n-2}+\cdots \pm p_{n} .
$$

Any quantity which satisfies the equation

$$
D(\lambda)=0
$$

and obeys the associative and commutative laws of algebra - whether it be a number or not-must also satisfy the relation

$$
\lambda^{n}=-p_{1} \lambda^{n-1}+p_{2} \lambda^{n-2}+\cdots \mp p_{n} ;
$$

and if we multiply this by $\lambda$ throughout and then eliminate $\lambda^{n}$ we get

$$
\lambda^{n+1}=\left(p_{1}^{2}+p_{2}\right) \lambda^{n-1}-\left(p_{1} p_{2}+p_{3}\right) \lambda^{n-2}+\cdots,
$$

which is of the form

$$
\lambda^{n+1}=p_{1}^{(n+1)} \lambda^{n-1}+p_{2}^{(n+1)} \lambda^{n-2}+\cdots .
$$

Similarly, by a continuation of the same process we may get a succession of equations, all of the form

$$
\lambda^{m}=p_{1}^{(m)} \lambda^{n-1}+p_{2}^{(m)} \lambda^{n-2}+\cdots .
$$

We call the typical polynomial on the right of (6) $f_{m}(\lambda)$ : graphically it represents a curve of degree $n-1$ passing through the $n$ points $-\lambda_{j},\left(-\lambda_{j}\right)^{m}$. But we do not wish to emphasize this geometric interpretation but rather the formal algebraic fact that our derivation has required only the elementary rules of algebra and the relation (5), and that when these rules are satisfied

$$
\lambda^{m}=f_{m}(\lambda) .
$$

Suppose, now, that we expand the quotient $f_{m}(\lambda) / D(\lambda)$ in partial fractions. The result is

$$
\frac{f_{m}(\lambda)}{D(\lambda)} \equiv \sum_{j=1}^{n} \frac{f_{m}\left(-\lambda_{j}\right)}{\lambda+\lambda_{j}} \frac{1}{\prod_{k \neq j}\left(-\lambda_{j}+\lambda_{k}\right)} .
$$

${ }^{3}$ The unconventional pecularities of sign in (1) and in (4) below happen to be convenient for our purposes later on. 
But $f_{m}\left(-\lambda_{j}\right)=\left(-\lambda_{j}\right)^{m}$ by $(7)$, and $D(\lambda)=\Pi\left(\lambda+\lambda_{k}\right)$ by (1). Hence

$$
f_{m}(\lambda) \equiv \sum_{j=1}^{n}(-\lambda)^{m} \prod_{k \neq j}\left(\frac{\lambda_{k}+\lambda}{\lambda_{k}-\lambda_{j}}\right) \text {. }
$$

Now (9) is an algebraic identity, and though we have used the process of division in setting it up, it does not require division by $\lambda$ as a process of verification. Hence it is again true that if $\lambda$ is any quantity which obeys the distributive and associative laws, such for example as a differential operator, and which satisfies (5),

$$
\lambda^{m} \equiv \sum_{j=1}^{n}\left(-\lambda_{j}\right)^{m} \pi_{j}^{\prime}(\lambda)
$$

where

$$
\pi_{j}^{\prime}(\lambda)=\prod_{k \neq j}\left(\frac{\lambda_{k}+\lambda}{\lambda_{k}-\lambda_{j}}\right) .
$$

Note that the quantities denoted by $\pi_{j}(\lambda)$ are polynomials of degree $n-1$ in $\lambda$ and are independent of $m$.

4. Matrices. We next observe that, though matrix multiplication is not in general commutative, it is so if we restrict ourselves to certain groups. In particular, if we begin with the unit matrix $I$, any other matrix $M$, and all scalar quantities (i.e., numbers), then all matrices which can be formed from these by a finite number of additions or multiplications are commutative. For obviously $M$ is commutative with itself and its powers, and with $I$, and with scalars, which observations together with the associative law are sufficient to warrant the general statement.

Furthermore, we know from the Hamilton-Cayley Theorem ${ }^{4}$ that

$$
D(M)=0 \text {, }
$$

where $D(\lambda)$ represents, as in $\S 3$, the characteristic function of $M$. In other words, $M$ satisfies all the requirements imposed upon $\lambda$ in deriving the identity (10), whence we conclude that

$$
M^{m}{ }_{.} \equiv \sum \mathbb{l}\left(-\lambda_{j}\right)^{m} \pi^{\prime}(M),
$$

where $\pi_{i}^{\prime}(M)$ is a matrix independent of $m$.

As a final step, we multiply this equation throughout by an arbitrary matrix $K$-which need not be commutative with the rest, since we shall perform no further operations-thus obtaining

$$
M^{m} K \equiv \sum\left(-\lambda_{j}\right) \pi_{j}(M)
$$

where $\pi_{j}(M)=\pi_{i}^{\prime}(M) K$ is again independent of $m$.

This is the fundamental identity upon which Duncan and Collar rely for their method. It is equivalent to $n^{2}$ equations of similar form connecting corresponding elements in the various matrices. For example, if $\alpha_{m}$ is written for the element in the $i$-th row and $j$-th column of $M^{m} K$, and $e_{j}$ for the correspondingly placed element in $\pi_{j}(M)$, it must be true that

$$
\alpha_{m}=e_{1}\left(-\lambda_{1}\right)^{m}+e_{2}\left(-\lambda_{2}\right)^{m}+\cdots+e_{n}\left(-\lambda_{n}\right)^{m} .
$$

- M. Bocher, Introduction to higher algebra, MacMillan, New York, 1929, p. 296. 
We again recall that $-\lambda_{j}$ is a root of the characteristic equation of $M$-that is, of the polynomial $D(\lambda)$-and hence is a number. The set $-\lambda_{j}$ are, in fact, just the roots which we wish to obtain. Similarly, the $e_{j}^{j}$ 's are numbers independent of $m$. But $\alpha_{m}$ is a numerical function of $m$.

5. The roots. Suppose now, that one of the roots which we will call $-\lambda_{1}$, is larger in absolute value than all the rest. Then if we select corresponding elements $\alpha_{m}$ and $\alpha_{m+1}$ from two consecutive orders of $M^{m} K$ we will have

$$
\frac{\alpha_{m+1}}{\alpha_{m}}=-\lambda_{1} \frac{1+\frac{e_{2}}{e_{1}}\left(\frac{\lambda_{2}}{\lambda_{1}}\right)^{m+1}+\frac{e_{3}}{e_{1}}\left(\frac{\lambda_{3}}{\lambda_{1}}\right)^{m+1}+\cdots}{1+\frac{e_{2}}{e_{1}}\left(\frac{\lambda_{2}}{\lambda_{1}}\right)^{m}+\frac{e_{3}}{e_{1}}\left(\frac{\lambda_{3}}{\lambda_{1}}\right)^{m}+\cdots},
$$

and hence obviously

$$
\lim _{m \rightarrow \infty} \frac{\alpha_{m+1}}{\alpha_{m}}=-\lambda_{1}
$$

In other words: if an arbitrary matrix $K$ is multiplied repeatedly by $M$, and if its characteristic equation has a largest root, then the ratio of corresponding elements in two consecutive products approaches this largest root as a limit as $m \rightarrow \infty$.

Similarly, we readily find that

$$
\left|\begin{array}{ll}
\alpha_{m+1} & \alpha_{m} \\
\alpha_{m} & \alpha_{m-1}
\end{array}\right|=\left(-\lambda_{1}\right)^{m}\left(-\lambda_{2}\right)^{m} \sum_{i \neq j} e_{i} e_{j}\left(\frac{\lambda_{i} \lambda_{j}}{\lambda_{1} \lambda_{2}}\right)^{m}\left(\sqrt{\overline{\lambda_{i}}}-\sqrt{\overline{\lambda_{j}}}\right)^{2} ;
$$

whence if $\left|\lambda_{1}\right|$ and $\left|\lambda_{2}\right|$ are greater than all other $\mid \lambda$ 's $\mid$, (whether they are themselves equal or not), we again have

$$
\lim _{m \rightarrow \infty} \frac{\left|\begin{array}{ll}
\alpha_{m+1} & \alpha_{m} \\
\alpha_{m} & \alpha_{m-1}
\end{array}\right|}{\left|\begin{array}{ll}
\alpha_{m} & \alpha_{m-1} \\
\alpha_{m-1} & \alpha_{m-2}
\end{array}\right|}=\left(-\lambda_{1}\right)\left(-\lambda_{2}\right) .
$$

In the same way it can be shown ${ }^{5}$ that provided $\left|\lambda_{1}\right|, \cdots,\left|\lambda_{i}\right|$ are all greater than $\left|\lambda_{i+1}\right| \cdots\left|\lambda_{n}\right|$

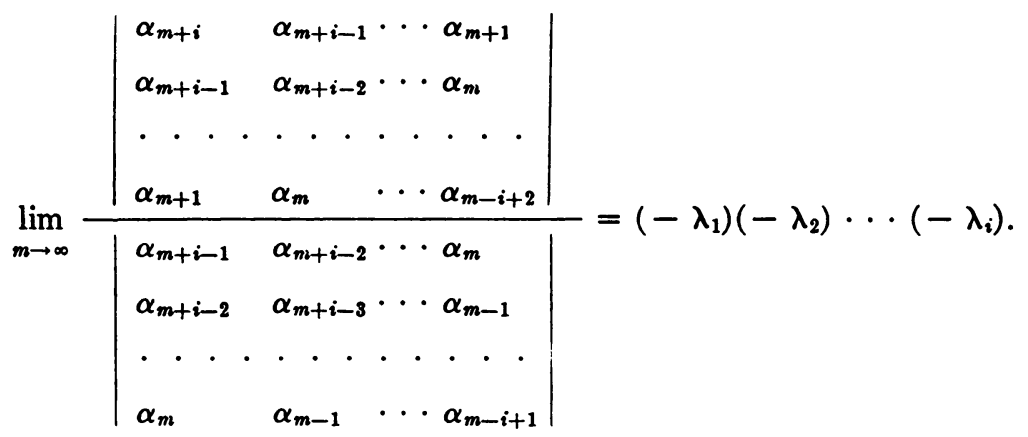

5 A. C. Aitken, Proc. Royal Soc. Edinburgh 46, 289-305 (1926), obtains formulae equivalent to these in a discussion of Bernoulli's method. 
These equations are sufficient to determine all the roots in the particular case where

$$
\left|\lambda_{1}\right|>\left|\lambda_{2}\right|>\left|\lambda_{3}\right| \cdots>\left|\lambda_{n}\right| \text {. }
$$

6. Example. As a simple example we may take

$$
M=\left\|\begin{array}{ll}
3 & 1 \\
2 & 2
\end{array}\right\|, \quad K=\left\|\begin{array}{l}
1 \\
0
\end{array}\right\|
$$

in which case

$$
\begin{array}{ll}
M K & =\left\|\begin{array}{l}
3 \\
2
\end{array}\right\|, \quad M^{2} K=\left\|\begin{array}{l}
11 \\
10
\end{array}\right\|, \quad M^{3} K=\left\|\begin{array}{l}
43 \\
42
\end{array}\right\|, \\
M^{4} K & =\left\|\begin{array}{l}
171 \\
170
\end{array}\right\|, \quad M^{5} K=\left\|\begin{array}{l}
683 \\
682
\end{array}\right\| .
\end{array}
$$

Taking the ratios of the first elements of consecutive matrices we get as the successive approximations to $-|\lambda|$,

$$
11 / 3=3.667, \quad 43 / 11=3.909, \quad 171 / 43=3.977, \quad 683 / 171=3.992 .
$$

Similarly we find that

$$
\frac{\left|\begin{array}{rr}
171 & 43 \\
43 & 11
\end{array}\right|}{\left|\begin{array}{rr}
43 & 11 \\
11 & 3
\end{array}\right|}=4 \text { and } \frac{\left|\begin{array}{rr}
683 & 171 \\
171 & 43
\end{array}\right|}{\left|\begin{array}{rr}
171 & 43 \\
43 & 11
\end{array}\right|}=4,
$$

which should be the product of $\lambda_{1}$ and $\lambda_{2}$.

The characteristic equation in this case is, however,

$$
D(\lambda)=\left|\begin{array}{cc}
\lambda+3 & 1 \\
2 & \lambda+2
\end{array}\right|=\lambda^{2}+5 \lambda+4,
$$

and its roots are -1 and -4 . The approximation is obvious.

7. Complex roots. So far we have considered only real roots: for obviously, since complex roots occur in conjugate pairs (the coefficients being assumed to be real) there can be no largest one. Suppose, then, that $\left|\lambda_{2}\right|=\left|\lambda_{1}\right|$ and that all other roots are smaller in absolute value. Then by (17),

$$
\lambda_{1} \lambda_{2}=\lim _{m \rightarrow \infty} \frac{\left|\begin{array}{cc}
\alpha_{m+1} & \alpha_{m} \\
\alpha_{m} & \alpha_{m-1}
\end{array}\right|}{\left|\begin{array}{cc}
\alpha_{m} & \alpha_{m-1} \\
\alpha_{m-1} & \alpha_{m-2}
\end{array}\right|} .
$$

This gives us the absolute value of the roots. It does not, however, determine the angles. To get this, we can best return to equation (14) and write (retaining only the leading terms)

$$
\alpha_{m}=e_{1}\left(-\lambda_{1}\right)^{m}+e_{2}\left(-\lambda_{2}\right)^{m} .
$$


Writing the similar equations for $m-1$ and $m+1$, and eliminating $e_{1}$ and $e_{2}$, we get

$$
\lambda_{1} \lambda_{2} \alpha_{m-1}+\left(\lambda_{1}+\lambda_{2}\right) \alpha_{m}+\alpha_{m+1}=0 .
$$

Substituting the value of $\lambda_{1} \lambda_{2}$ as given by (19) we get finally

$$
-\left(\lambda_{1}+\lambda_{2}\right)=\lim _{m \rightarrow \infty} \frac{\left|\begin{array}{cc}
\alpha_{m+1} & \alpha_{m-1} \\
\alpha_{m} & \alpha_{m-2}
\end{array}\right|}{\left|\begin{array}{cc}
\alpha_{m} & \alpha_{m-1} \\
\alpha_{m-1} & \alpha_{m-2}
\end{array}\right|} .
$$

This, together with (19) is sufficient to determine the pair of roots.

As written, the formula applies even if the roots are real. ${ }^{6}$ When they are complex it is best to write $-\lambda_{1}=-\lambda_{2}=\rho e^{i \phi}$. Then obviously we need only replace the $\lambda_{1} \lambda_{2}$ of (19) by $\rho^{2}$, and the $-\left(\lambda_{1}+\lambda_{2}\right)$ of (20) by $2 \rho \cos \phi$.

Similar, but more complicated, formulae can be obtained when more than two roots have the same absolute value.

8. The method of Daniel Bernoulli. We now note that any polynomial in $\lambda$, which we take in the form

$$
D(\lambda)=\lambda^{n}+p_{1} \lambda^{n-1}-p_{2} \lambda^{n-2}+\cdots \mp p_{n-1} \lambda \pm p_{n}
$$

as before, can be written as

$$
D(\lambda)=\left|\begin{array}{cccccc}
\lambda & 1 & 0 & \cdots & 0 & 0 \\
0 & \lambda & 1 & \cdots & 0 & 0 \\
0 & 0 & \lambda & \cdots & 0 & 0 \\
\cdots & \ldots & \ldots & \cdots & . & \ldots \\
0 & 0 & 0 & \cdots & \lambda & 1 \\
p_{n} & p_{n-1} & p_{n-2} & \cdots & p_{2} & p_{1}+\lambda
\end{array}\right| .
$$

But this is the characteristic function of the matrix

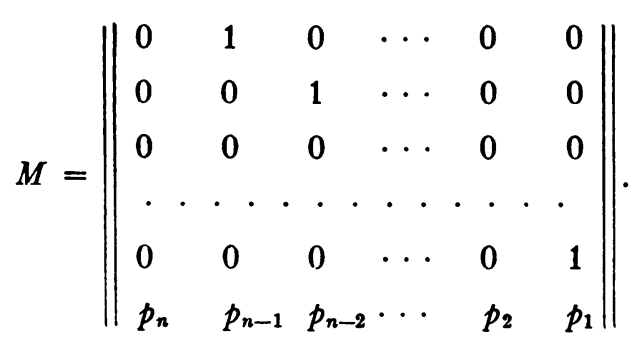

Hence if we choose for $K$ any matrix whatever, we may solve for the largest roots by any of the equations of $\S \S 4$ and 6 .

It is particularly convenient to take $K$ in the form

\footnotetext{
- It is not even necessary that they be equal in absolute value, though unless they are equal (or nearly equal) (15) will obviously be a more convenient formula.
} 


$$
K=\left\|\begin{array}{ccccc}
0 & 0 & 0 & \cdots & \alpha_{0} \\
0 & 0 & 0 & \cdots & \alpha_{1} \\
0 & 0 & 0 & \cdots & \alpha_{2} \\
\cdot & \cdot & \cdot & \cdots & \cdot \\
0 & 0 & 0 & \cdots & \alpha_{n-1}
\end{array}\right\|
$$

Then we have

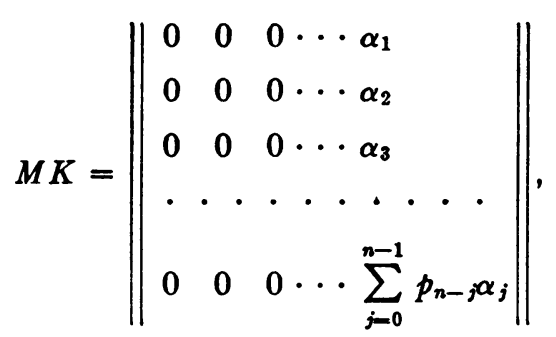

which is again of the same form as $K$. If we denote $\sum_{j=0}^{n-1} p_{j+1} \alpha_{n-j-1}$ by $\alpha_{n}$, we also have

And in general

$$
M^{2} K=\left\|\begin{array}{ccccc}
0 & 0 & 0 & \cdots & \alpha_{2} \\
0 & 0 & 0 & \cdots & \alpha_{3} \\
0 & 0 & 0 & \cdots & \alpha_{4} \\
\cdots & \ldots & \ldots & \ldots & \ldots \\
0 & 0 & 0 & \cdots & \sum_{i=0}^{n-1} p_{i-1} \alpha_{n-i}
\end{array}\right\|
$$

where

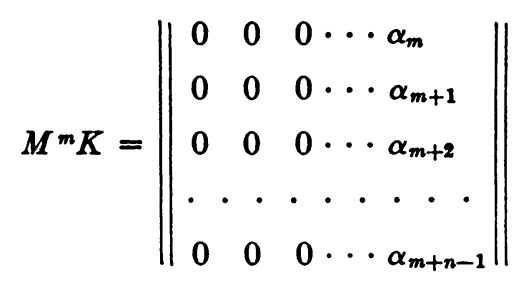

$$
\alpha_{k+1}=\sum_{j=0}^{n-1} p_{j+1} \alpha_{k-j}, \quad k>n-2 .
$$

This entire set of matrices, however, is characterized by a simple sequence of $\alpha$ 's, of which the defining equation is (24). Obviously, it is also true that any set of four consecutive $\alpha$ 's in this sequence also constitutes a set of corresponding elements from four consecutive matrices of the set $M^{m} K$. Hence, the use of the symbol $\alpha$ in this connection is consistent with its use in $\$ \$ 3-6$. But (24) is the recursion formula used in Bernoulli's method of solution as developed by Euler, Lagrange and Aitken. Hence this particular special case of the results of Duncan and Collar is identical with Bernoulli's method.

Concerning this method Whittaker and Robinson 7 say: "Though hardly now of first-rate importance, it is interesting and worthy of mention." Our tests at the

${ }^{7}$ E. T. Whittaker and G. Robinson, Calculus of observations, 2nd ed., Blackie \& Son, London, 1929. 
Laboratories, however, have shown it as good as any other method in the case of complex roots. Such inferiority as it may have compared to the root-squaring method as regards speed is quite compensated by the fact that it is self-correcting: that is, an error at any stage of the process merely prolongs the calculations, but does not invalidate it.

9. The method of R. L. Dietzold. Another form into which the general results of Duncan and Collar can be thrown is obtained by using the conjugate form of (21) together with the same matrix for $K$ as before. Denoting the conjugate of $M$ by $M^{\prime}$, we have from (22) and (23)

If, then, we define

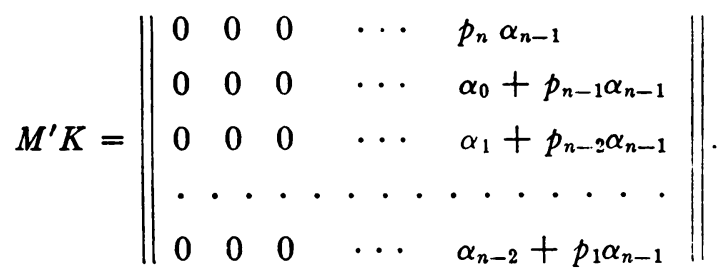

$$
\alpha_{0}^{\prime}=p_{n} \alpha_{n-1}, \quad \alpha_{j}^{\prime}=\alpha_{j-1}+p_{n-j} \alpha_{n-1},
$$

$M^{\prime} K$ becomes identical with (23), except that all the $\alpha$ 's are primed. In general, if we set

$$
\alpha_{j}^{(m)}=\alpha_{j-1}^{(m-1)}+p_{n-j} \alpha_{n-1}^{(m-1)},
$$

and understand that $\alpha_{-1}^{(m)}$ is zero for all $m$, we have

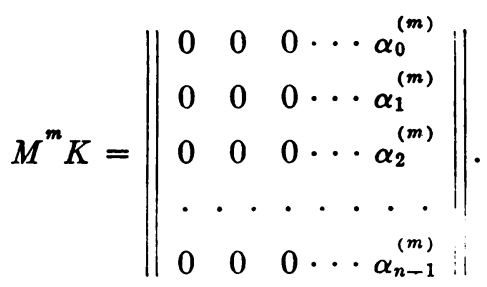

In this case, as in all others, the index $m$ is the one which is to be varied in using formulae such as (16)-(20).

This variant of the general scheme of Duncan and Collar was developed by $\mathrm{Mr}$. R. L. Dietzold of the Bell Telephone Laboratories. but has not been published. As compared with Bernoulli's, it has the merit of using a large number of simple operations instead of a small number of complicated ones. It is approximately as fast, and like all schemes based on Duncan and Collar's results, it is self-correcting.

10. The method of Graeffe. There is also a closi connection between Duncan and Collar's processes and the root-squaring method. This method, which is usually attributed to Graeffe, seems actually to have been developed first by Dandelin, and has had the attention of a long list of mathematicians, including Lobachevski, Encke, Brodetsky and Smead, and Hutchinson.

This connection can best be established ${ }^{8}$ by recalling that the roots $-\lambda_{j}$ of the

${ }^{8}$ M. Bocher, Introduction to higher algebra, MacMillan, New York, 1929, p. 283, Theorem 3. 
matrix $\lambda I+M$ are invariant under transformations of the type $T^{-1}[\lambda I+M] T$. Furthermore, it is possible to find a transformation of this sort which will throw $M$ in to the form

$$
M^{*}=T^{-1} M T=\left\|\begin{array}{cccc}
\lambda_{1} & 0 & \cdots & 0 \\
0 & \lambda_{2} & \cdots & 0 \\
\cdot & \cdots & \cdots & . \\
0 & 0 & \cdots & \lambda_{n}
\end{array}\right\| \text {, }
$$

and hence $\lambda I+M$ into the form $\lambda I+M^{*}$, since $T^{-1} I T$ is obviously $I$.

This same transformation, however, carries $M^{m}$ into $M^{* m}$, as we readily see from the identity

$$
\begin{aligned}
T^{-1} M^{m} T & \equiv T^{-1}\left(M T T^{-1} M T \cdots T^{-1} M\right) T \\
& \equiv\left(T^{-1} M T\right)\left(T^{-1} M T\right) \cdots\left(T^{-1} M T\right) \\
& \equiv M^{* m} .
\end{aligned}
$$

Hence the characteristic equations of $M^{m}$ and $M^{* m}$ must also have identical roots. But, obviously,

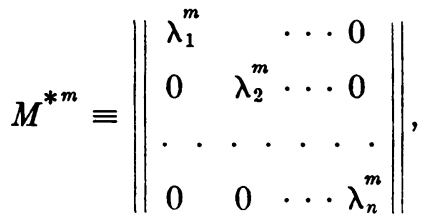

so that the roots of its characteristic equation, and therefore also those of the characteristic equation of $M^{m}$, must be $-\lambda_{j}^{m}$.

But if we take $K=I$ in Duncan and Collar's process of matrix iteration, the successive matrices obtained are $M^{m}$. Hence the whole process may be regarded as one which sets up a sequence of characteristic equations with roots $-\lambda_{j},-\lambda_{j}^{2}, \cdots$ and in general $-\lambda_{j}^{m}$.

In the root-squaring process as originally developed only the powers $-\lambda_{j}^{2},-\lambda_{j}^{4}$, $-\lambda_{j}^{8}, \ldots$ were obtained, which corresponds in matrix terms to getting first the product of $M$ by $M$, which is $M^{2}$; then the product of $M^{2}$ by $M^{2}$ which is $M^{4}$, and so on. Thus high powers are reached with a smaller number of matrix operations, which is theoretically desirable. Practically, however, the superiority is not so apparent. For the zeros of (22) are rapidly replaced by numbers in forming powers of $M$, so that a multiplication such as $M^{8} \cdot M^{8}$ involves many more arithmetical operations than a multiplication of the form $M \cdot M^{8}$. Furthermore, an error at any point of the root-squaring method perpetuates itself, whereas in the other method an error at any stage is merely equivalent to starting over again with a new value of $K$.

Our experience leads us to believe that the methods of $\$ \S 8$ and 9 are generally to be preferred, at least when computations are to be performed by a clerical staff of computers.

11. The method of Bernoulli as developed by Lagrange. There is also a very close connection between the iterated matrix $M^{m}$ and a development of Lagrange's which he characterizes as based upon that of Daniel Bernoulli. In it, he notes that 


$$
\frac{D^{\prime}(\lambda)}{D(\lambda)}=\sum \frac{1}{\lambda+\lambda_{j}}=\frac{n}{\lambda}+\frac{s_{1}}{\lambda^{2}}+\frac{s_{2}}{\lambda^{3}}+\frac{s_{3}}{\lambda^{4}}+\cdots,
$$

where

$$
s_{m}=\left(-\lambda_{1}\right)^{m}+\left(-\lambda_{2}\right)^{m}+\cdots+\left(-\lambda_{n}\right)^{m} ;
$$

and it is the quotient $s_{m} / s_{m-1}$ which Lagrange uses. Obviously, these are just the sums of the elements in the principal diagonals of $M^{* m}$. But Lagrange's method of obtaining them by dividing $D(\lambda)$ into its derivative is preferable. Besides, in spite of what might at first be assumed, it is self-correcting.

It is of historical interest to note that a very similar development was worked out by Legendre ${ }^{9}$ independently of Lagrange, and at about the same time. Both of these writers, however, knew of earlier work by Euler, who had carried out a similar development using instead of $D^{\prime}(\lambda)$ an arbitrary polynomial of degree $n-1$, which
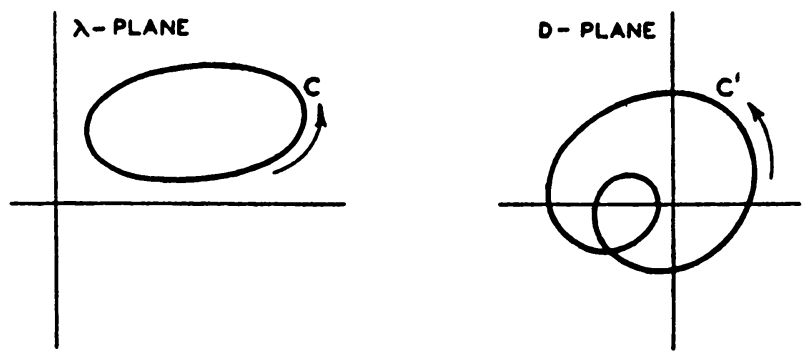

Fig. 1.

merely has the effect of replacing the $s_{m}$ 's in the right-hand member of (28) by the $\alpha_{m}$ 's defined by (14). In other words, the method of Euler was exactly equivalent to that of Duncan and Collar, except that in the former there was no obvious criterion for the choice of a convenient form of numerator, whereas it is easy to choose matrices $K$ which will lead to a simple succession of operations, as we have illustrated in Sections 8 and 9.

\section{PART II-CONFORMAL MAPPING}

12. The method of Routh. The second group of methods to which I wish to refer are all founded upon a well-known theorem of Cauchy. If we represent the complex variable $\lambda$ by one plane, and the complex variable $D$ by another, then the equation

$$
D(\lambda)=\lambda^{n}+p_{1} \lambda^{n-1}-p_{2} \lambda^{n-2}+\cdots \pm p_{n},
$$

may be looked upon as a transformation by means of which the $\lambda$-plane is mapped upon the $D$-plane. The correspondence between $\lambda$ and $D$, however, is not $1: 1$ but in general $n: 1$; and hence a simple closed curve $C$ in the $\lambda$-plane (Fig. 1) passes into a much more complicated curve $C^{\prime}$ in the $D$-plane. In regard to the curve $C^{\prime}$ the theorem in question says that the number of times it loops around the origin is exactly equal to the number of roots of $D(\lambda)=0$ which lie inside $C$.

9 Legendre's development was in terms of the reciprocal powers of the roots, instead of their direct powers. Otherwise the two were identical. 
This rule appears first to have been applied by Routh to the problem of determining the number of roots with positive real parts, a problem which interested him because of its relation to the stability of linear dynamical systems. For this purpose he used as the contour in the $\lambda$-plane the imaginary axis closed by a semicircle of infinite radius, thus enclosing the entire right half of the plane. For this particular contour he explained in great detail how from the sequence of intersections of $C^{\prime}$ with the real and imaginary axes the number of roots could be found without more definite information as to the shape of $C^{\prime}$. He also developed a sequence of functions, similar to Sturm functions, by means of which the number of roots could be determined from the polynomial directly without even knowing the real and imaginary intercepts of $C^{\prime}$. He did not extend either of these studies to the point of locating the roots more exactly, but both are capable of such extension and have actually been used.

13. The method of G. R. Stibitz. The second method-the one using functions similar to the Sturm functions-was developed further by G. R. Stibitz of the Bell Telephone Laboratories. He observes, first, that the method can also be used to find the number of roots with real parts greater than $\lambda_{0}$. To do this, it is merely necessary to replace $\lambda$ by $\lambda-\lambda_{0}$ in the polynomial (4), and then proceed as outlined by Routh. By carrying out this process for enough values of $\lambda_{0}$, the roots can be segregated within strips parallel to the imaginary axis. Then by a definite routine (resembling in its essentials the Weierstrass subdivision process in point-set theory) the real values of the roots can be found to any desired degree of approximation. When this has been accomplished, the imaginary parts are determined at once as a ratio of two of the Sturm-like functions.

Stibitz has developed complete schedules for the computations required in solving polynomials by this method, for all values of $n$ up to 10 . The method has been tried, and works reasonably well, though perhaps not as rapidly as those explained in Sections 8 and 9. I suspect that the decision in this case, however, must remain a conditional one; for the computational routine of Stibitz' method is complicated (i.e., varied) as compared with the extremely simple (i.e., repetitive) routines of Sections 8 and 9. For this reason, it is not as well adapted to use in an industrial computing laboratory. In the hands of a mathematician who thoroughly understood its theoretical origin it might show up much better.

14. The method of A. J. Kempner. Kempner's methods ${ }^{10}$ resemble more nearly the other portion of Routh's work. He chooses as his contour $C$ a circle of radius $r$ about the origin as center. Then $\lambda=r e^{i \theta}$, and (4) becomes

$$
\begin{aligned}
D(\lambda)= & {\left[r^{n} \cos n \theta+p_{1} r^{n-1} \cos (n-1) \theta-p_{2} r^{n-2} \cos (n-2) \theta+\cdots\right] } \\
& +i\left[r^{n} \sin n \theta+p_{1} r^{n-1} \sin (n-1) \theta-p_{2} r^{n-2} \sin (n-2) \theta+\cdots\right] .
\end{aligned}
$$

Thus the real and imaginary parts of $D$ are trigonometric sums, which, as Kempner remarks, could be calculated by means of a harmonic synthesizer, such for example as the Michelson "analyzer." Thus two curves would be obtained, one giving the real part of $D$, and the other its imaginary part, both as functions of $\theta$. From this point on, Kempner suggests two possible routines. First, to regard these curves as parametric representations of $D$, and from them construct the curve $C^{\prime}$ itself. Second, to keep them as separate curves and not bother further about $C^{\prime}$. In both cases, he

10 University of Colorado Studies 16, 75 (1928); Bulletin of the Amer. Math. Society 41, 809 (1935). 
develops rules very similar to Routh's for finding the number of roots directly from the sequence of intercepts with the axes.

He uses this routine to segregate the roots in annular rings, and then tracks down their absolute values by a suitably chosen succession of intermediate circles. The angle of any root is, of course, automatically determined as the value of $\theta$ at which the real and imaginary parts of (30) vanish when $r$ is given the particular value appropriate to that root.

Kempner also mentions the possibility of applying the method to sectorial instead of annular regions, but does not develop this idea to a significant degree.

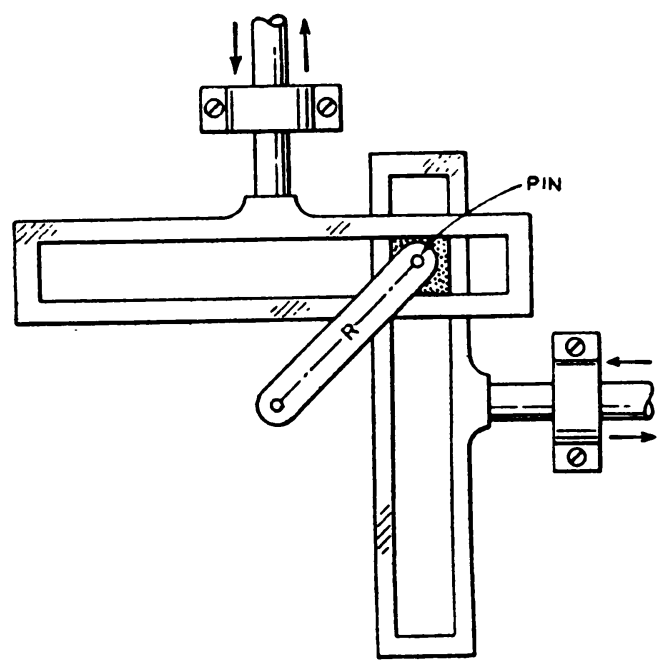

Fig. 2.

15. The isograph. Kempner's method was also developed independently, but somewhat later (1934) at Bell Telephone Laboratories, and led to the construction of a machine, called the isograph, which draws the curve $C^{\prime}$ corresponding to a circle of any radius $r$.

Since the independent variable in plotting the curves is an angle, what is required for the isograph is a rotating unit that provides two linear motions--one proportional to the sine and the other to the cosine of the angle. There would have to be ten of these units to provide for the ten variable terms of a tenth degree equation, and while the first unit moves through an angle $\theta$; the second unit must move through an angle $2 \theta$, the third unit through an angle $3 \theta$, and so on. Then by providing a means of summing the sine and cosine motions separately, and allowing these sums to control two perpendicular motions of a pencil and drawing board, a closed curve will be described as $\theta$ increased from 0 to 360 degrees.

To secure motions proportional to the sine and cosine of the angle of rotation, the isograph utilizes the "pin and slot" mechanism illustrated in Fig. 2. Here an arm rotating about a fixed point carries a pin arranged to slide, by means of a rectangular block, in rectangular slots cut in two slide-bars, each of which is free to move back and forth in one direction only - the two motions being at right angles to each other. These motions are equal to the length $R$ of the arm times the sine and cosine of the angle of rotation. 
The ten units provided are geared to a common driving motor, but the gearing is designed so that when the arm of the first unit moves through an angle $\theta$, that of the second unit will move through an angle $2 \theta$, that of the third through $3 \theta$, and so on.

To provide for summing up all the sine terms and all the cosine terms, the ends of all the slide-bars carry pulleys so that a single wire may be carried around all the sine pulleys and another around all the cosine pulleys as indicated in Fig. 3. Station-

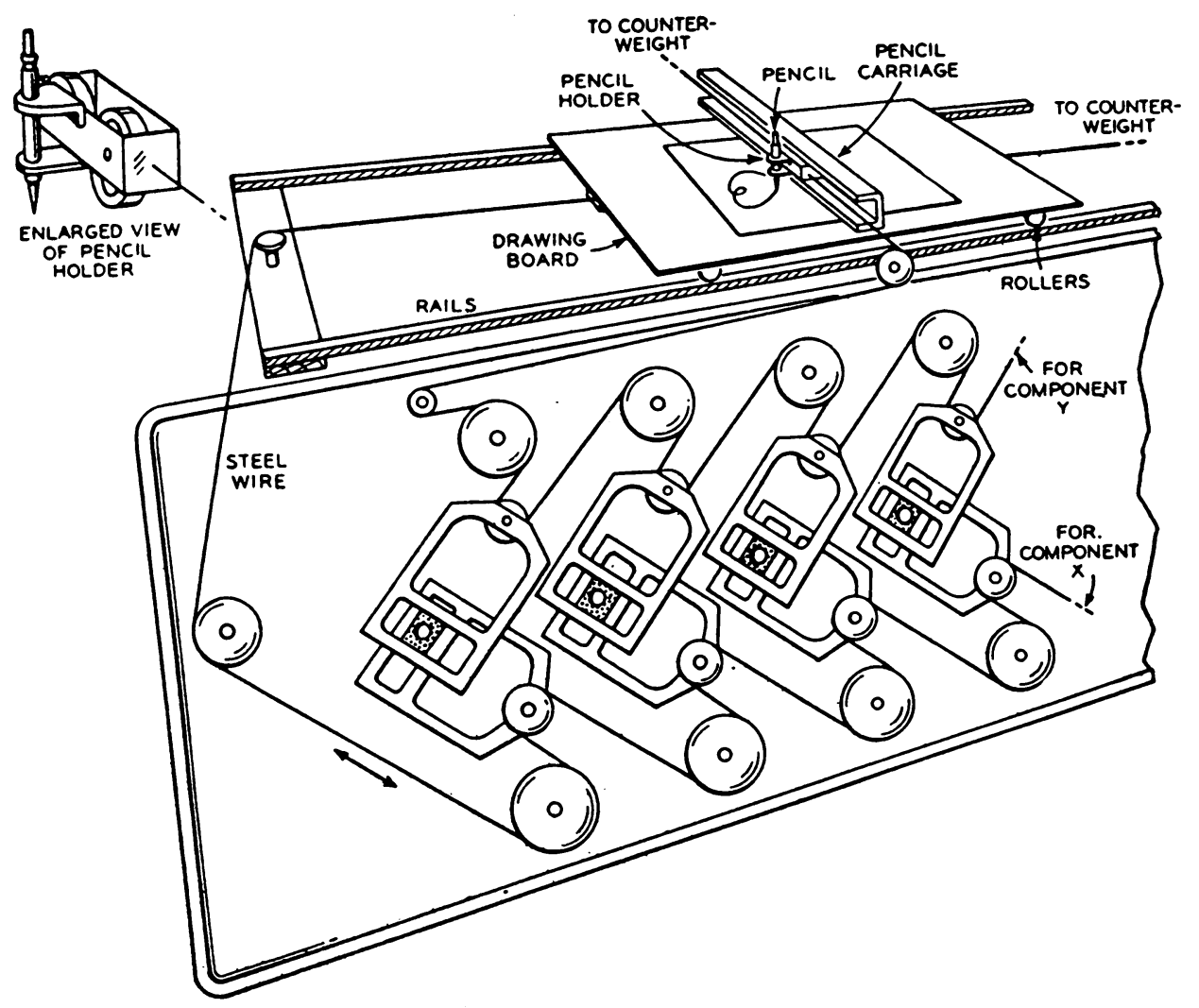

FIG. 3.

ary pulleys are mounted between the movable ones so as to keep the direction of pull on the wires in line with the motion of the slide-bars. These wires control the relative motions of a pencil and drawing board to plot a curve as the angle is varied from zero to three hundred and sixty degrees.

The construction of the rotating elements is shown in Fig. 4. The drive shaft passes through the bed plate and is fastened to the center of a steel bar that acts as the arm of Fig. 2. This bar is grooved to receive the pin of the "pin and slot" mechanism. In order that the pin may be adjusted for different crank lengths, corresponding to the coefficients $p_{k} r_{n-k}$ of the various terms in the equation, a rack is cut along one edge of the groove so that a pinion attached to the pin may move it along the bar. After adjustment the pin is secured in place by a set-screw.

The top of the bar carries a carefully graduated scale to which the center of the pin must be set accurately. The scale is made visible at the center of the pin by con- 
structing the latter as a hollow cylinder. A vernier scale within the cylinder enables the effective arm length to be adjusted very exactly to the desired value on either side of the center-one side for positive coefficients and the other for negative. The total range of adjustment is three inches.

The hollow pin turns in a rectangular bronze block which fits the slots of two slide bars, one for the sine motion and one for the cosine motion. The slide bars are identical steel plates running in bronze ways set accurately at right angles to each

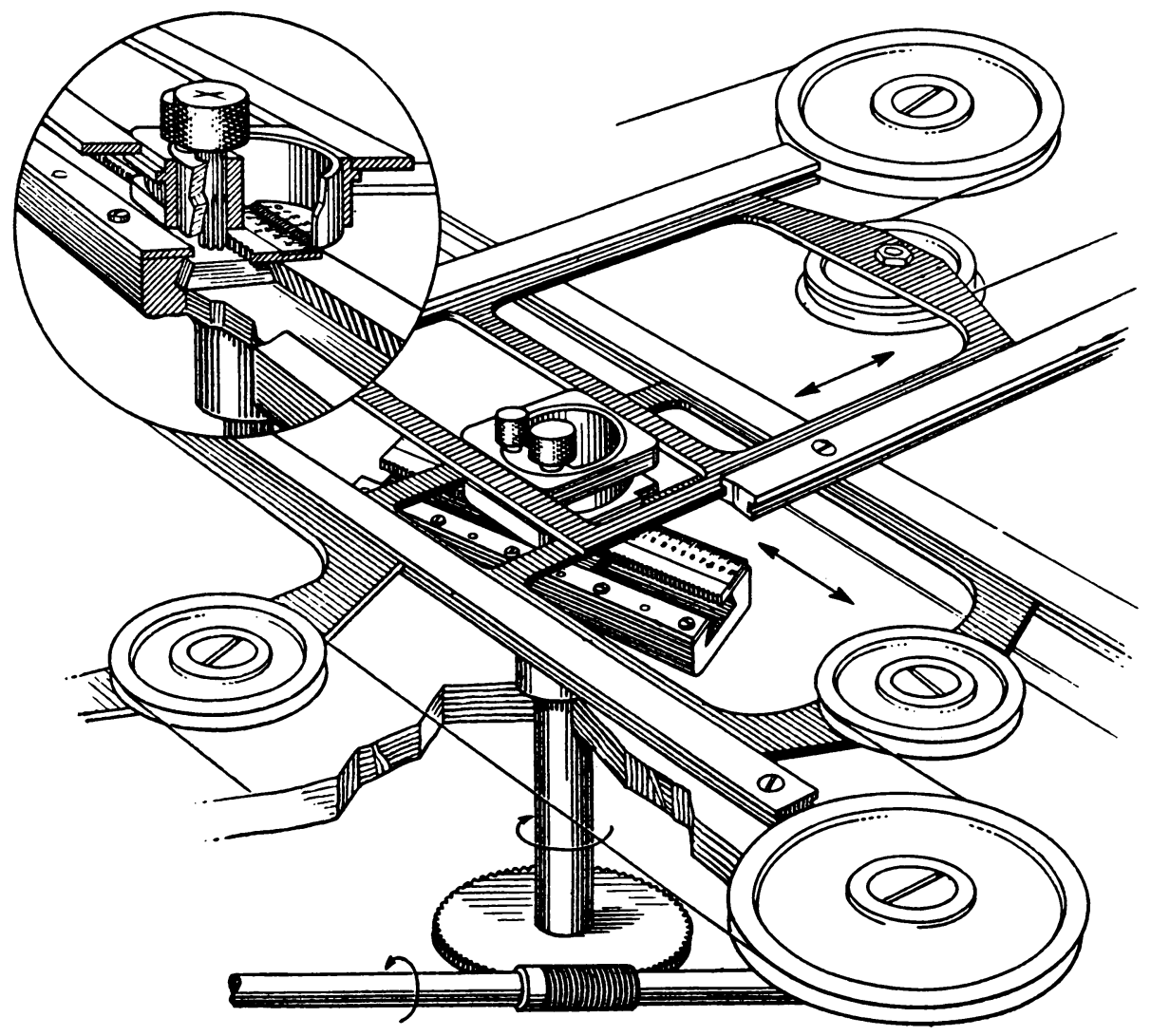

Fig. 4.

other. At the end opposite to the slot each plate carries a pulley around which is passed the wire that sums up the sine or cosine motions of the ten elements. One end of each wire is fixed. The other end of the cosine wire is led by pulleys to the drawing board, which consists of a thin aluminum sheet mounted on ball-bearing rollers so that it is free to move back and forth in only one direction. A counterweight fastened to the other edge of the board keeps the wire under constant tension. The free end of the sine wire is led by pulleys to a counterweighted pencil carriage, which is mounted with ball bearings in a fixed guide crossing the drawing board at right angles to its direction of motion. Thus the board is displaced back and forth in proportion to the sum of the cosine terms, and the pencil is displaced back and forth 
in a perpendicular direction in proportion to the sum of the sine terms; and this combined motion gives the desired curve.

In operation, the isograph has given accuracies of one per cent or better: and of course gives them quite rapidly. In fact, the most rapid method we have at present is that of using the isograph to obtain this degree of accuracy, and then improving it either by the methods explained in $\$ \S 8$ and 9 , or by successive approximation to the quadratic factors.

16. Conclusion. In conclusion I wish merely to point out that in none of the methods which I have described is computation with complex numbers involved. They are all real methods. At present this seems to be a fundamental requirement imposed upon us by commercial computing machines, since the multiplication of two complex numbers on such machines requires six, and division eight, separate operations. If this restriction were removed, other methods might conceivably prove to be more rapid.

Partly with this in mind, and partly because we must frequently deal with complex quantities in other connections, we are at present developing a computing machine for complex quantities. When it is completed, as we hope it will be in the course of the present year, we shall undertake a further study of methods which now are clearly ruled out by mechanical limitations.*

\section{POSTSCRIPT BY R. L. DIETZOLD}

When the foregoing paper was written, it was intended for immediate publication. By coincidence, however, several other papers of similar character appeared at just about that time, and Dr. Fry concluded that the subject was of too limited interest to justify publishing another.

Since then, the situation has changed in several ways. First, the interest in methods of numerical computation has greatly increased, largely because war activities have led to much work of that kind. Second, the specific problem of root-finding has become a live one because its fundamental importance in linear dynamics is more widely recognized. Finally, a few new methods of iteration have been evolved and some new types of computing machines developed. The paper therefore now has a timeliness which it lacked when written, but a few comments are required to bring it up to date. The most important of these are noted in the following paragraphs.

In the Bell Telephone Laboratories the available computing equipment has been materially improved through the development of the relay computer by Stibitz and this inevitably reacts upon the relative convenience of various methods of solution. Although the relay computer is very flexible in respect to the type of problem it can handle, it is particularly well suited to iterative processes such as Bernoulli's method of root extraction; for once the proper instructions have been set into the control tape which governs the machine, all successive operations are performed without further supervision. The simplicity of Bernoulli's rule, which requires only that the machine accumulate $n-1$ of the $\alpha$ 's, each multiplied by the appropriate coefficient from the polynomial, recommends it for mechanization. The instructions are easily

* This machine was placed in service in 1940 and was demonstrated at the summer meeting of the American Mathematical Society in Hanover, New Hampshire in September of that year. The relay computers referred to in Dr. Dietzold's postscript are still more versatile devices which have been developed since that time. 
set up, and the machine is not required to recall very many numbers at any stage in the process. Bernoulli's method is likewise well adapted to computing equipment of the punched-card type, provided only that the accumulator is designed to recognize algebraic sign.

One of the routines which may be set up in a relay computer enables the algebraic operations to be performed on complex numbers with the ease that the same operations are performed on real numbers with a mechanical computing machine. The availability of this aid makes Newton's method useful for root improvement in the complex domain, and some on the Laboratories' computing staff prefer it to Bairstow's method, although the margin of choice is not great.

Bairstow's variation of Newton's method avoids computation with complex quantities by improving the coefficients of a trial quadratic factor. The trial factor, say $Q(\lambda)=\lambda^{2}+a \lambda+b$, is divided twice in to the polynomial, and the rates of change of the remainder coefficients found from the second remainder, as in Horner's process. The method has by now been sufficiently publicized ${ }^{11}$ nevertheless, it can be given here, since it is short to state. The polynomial being expressed as

$$
D(\lambda)=\left(r_{0} \lambda+s_{0}\right)+Q(\lambda)\left(r_{1} \lambda+s_{1}\right)+Q^{2}(\lambda)\left(t_{0}+t_{1} \lambda+\cdots\right),
$$

improved coefficients for $Q$ are

$$
a^{\prime}=a-\frac{\left|\begin{array}{ll}
r_{0} & r_{1} \\
s_{0} & s_{1}
\end{array}\right|}{\mid \begin{array}{ll}
a r_{1}-s_{1} & r_{1} \\
b r_{1} & s_{1}
\end{array}}, \quad b^{\prime}=b+\frac{\left|\begin{array}{ll}
a r_{1}-s_{1} & r_{0} \\
b r_{1} & s_{0}
\end{array}\right|}{\left|\begin{array}{ll}
a r_{1}-s_{1} & r_{1} \\
b r_{1} & s_{1}
\end{array}\right|} .
$$

Newton's method typifies a class which is deliberately excepted from treatment in Fry's paper; methods in this class are characterized by the property that only sometimes do they lead to a solution. Newton's method, for example, can never lead to a complex root if the iterative process is started from a real trial value. Bairstow's method has a similarly restricted region of convergence and was, quite properly, advanced by him only as a means for improving roots already located approximately.

Methods which sometimes fail to converge may still be very useful if, in application, they converge of ten enough and fast enough. Newton's method and its variations, however, almost always fail unless they can be started from values closely corresponding to roots. But in 1941, Shih-Nge Lin revealed an algorith ${ }^{12}$ remarkable

${ }^{11}$ Bairstow gave the method only in Reports and Memoranda No. 154, Advisory Committee for Aeronautics, Oct., 1914 (H. M. Stationer's Office), but it was made generally available by Frazer and Duncan, Proc. Royal Soc. London 125, 68-82 (1929). Hitchcock offered the method as An improvement on the G.C.D. method for complex roots, Jour. Math. Phys. 23, 69-74 (1944). Hitchcock proposes that the roots be improved by this method af ter only approximate location by the G.C.D. method, which he gave in Jour. Math. Phys. 17, 55-58 (1938). The G.C.D. method is nearly identical with the method of G. R. Stibitz, described by Fry. Bairstow's method was also rediscovered by Friedman, whose work is noted in Bull. Amer. Math. Soc. 49, 859-860 (1943). Bairstow's formulae give the leading terms of series developments of the coefficients by Fry, who concluded, after an investigation of the convergence, that the expansion was suitable only for root-improvement.

${ }^{12} \mathrm{~A}$ method of successive approximations of evaluating the real and complex roots of cubic and higher order equations, Jour. Math. Phys. 20, 153 (1941). 
both for simplicity and convergence. By Lin's method, the polynomial is divided only once by a trial quadratic factor; if ${ }^{13}$

$$
\begin{aligned}
D(\lambda) & \equiv p_{0}+p_{1} \lambda+p_{2} \lambda^{2}+\cdots \\
& =\left(r_{0} \lambda+s_{0}\right)+Q(\lambda)\left(q_{0}+q_{1} \lambda+q_{2} \lambda^{2}+\cdots\right),
\end{aligned}
$$

improved coefficients for $Q$ are

$$
a^{\prime}=\frac{q_{0} p_{1}-q_{1} p_{0}}{q_{0}^{2}}, \quad b^{\prime}=\frac{p_{0}}{q_{0}} .
$$

If it converges, the process determines the factor corresponding to the roots of least absolute value; thus a suitable initial choice for $Q$ is

$$
\lambda^{2}+\left(p_{1} / p_{2}\right) \lambda+\left(p_{0} / p_{2}\right)
$$

In application, the process does very often converge, although sometimes slowly. When the convergence of Lin's method is slow, Bairstow's method offers a valuable supplement. Lin's method is used until the size of the remainder indicates that an approximation to a quadratic factor has been obtained; Bairstow's process, started from a sufficiently close approximation, will converge, and when it converges, it converges rapidly.

The combination of these two methods provides useful, and usually adequate, equipment for the work-a-day solution of polynomial equations. In recalcitrant cases, mechanical aids are particularly helpful. Bernoulli's method is always available, but is quite likely to be slow in cases for which Lin's method has already failed. This makes little difference if the iterative process is performed automatically by a relay computer, but recommends devices to accelerate the convergence if the computation must be performed without aid. An efficient device for accomplishing this is given by A. C. Aitken in a very full discussion ${ }^{14}$ of numerical methods for evaluating the latent roots of matrices.

Like most of those who use matrix methods, Aitken is concerned not solely with the solution of polynomial equations, but rather with the more general problem of determining the characteristic roots (and also the characteristic vectors) of matrices. Preliminary reduction of the matrix to the rational canonical form involves so many operations, ${ }^{15}$ that one would commonly start the general problem with a matrix $M$ having few vanishing elements. In this event, we lose one of the reasons for preferring Bernoulli's method (i.e., repeated multiplication by $M$ ) to matrix powering by the root-squaring method, for the latter method arrives at high powers of $M$ with fewer operations, thus providing another means for hastening the convergence. The advantage is, however, partly illusory except for the limited class of computers who are so unerring that they can afford to sacrifice the self-correcting feature of the former procedure.

\footnotetext{
${ }^{13}$ A departure from Fry's notation is convenient here.

14 Proc. Royal Soc. Edinburgh 57, 172-181 (1937).

${ }^{15}$ Harold Wayland, Expansion of determinantal equations into polynomial form, Quarterly Appl.
} Math. 2, 277-306 (1945). 